\title{
Hybrid integral transforms analysis of the bioheat equation with variable properties
}

\author{
Renato M. Cotta ${ }^{\mathrm{a}, *}$, Bianca P. Cotta ${ }^{\mathrm{b}}$, Carolina P. Naveira-Cotta ${ }^{\mathrm{a}}$, Gerson Cotta-Pereira ${ }^{\mathrm{c}}$ \\ ${ }^{a}$ LTTC - Laboratory of Transmission and Technology of Heat, Mechanical Engineering Department - Escola Politécnica E' COPPE, \\ Universidade Federal do Rio de Janeiro, UFRJ, Cx. Postal 68503 - Cidade Universitária, 21945-970 Rio de Janeiro, RJ, Brazil \\ ${ }^{\mathrm{b}}$ Dermatology - HUAP - Hospital Universitário Antônio Pedro UFF - Universidade Federal Fluminense, Niteroi, RJ, Brazil \\ ' 3 a. Enfermaria - Serviço de Imunoquímica e Histoquímica, Santa Casa de Misericórdia, Rio de Janeiro, Brazil
}

\section{A R T I C L E I N F O}

\section{Article history:}

Received 18 March 2010

Received in revised form

21 April 2010

Accepted 22 April 2010

Available online 2 June 2010

\section{Keywords:}

Bioheat transfer

Pennes' equation

Heat conduction

Integral transforms

Heterogeneous media

\begin{abstract}
A B S T R A C T
Pennes' equation is the most frequently employed model to describe heat transfer processes within living tissues, with numerous applications in clinical diagnostics and thermal treatments. A number of analytical solutions were provided in the literature that represent the temperature distribution across tissue structures, but considering simplifying assumptions such as uniform and linear thermophysical properties and blood perfusion rates. The present work thus advances such analysis path by considering a heterogeneous medium formulation that allows for spatially variable parameters across the tissue thickness. Besides, the eventual variation of blood perfusion rates with temperature is also accounted for in the proposed model. The Generalized Integral Transform Technique (GITT) is employed to yield a hybrid numerical-analytical solution of the bioheat model in heterogeneous media, which reduces to the exact solution obtained via the Classical Integral Transform Method for a linear formulation with uniform coefficients. The open source UNIT code ("UNified Integral Transforms") is utilized to obtain numerical results for a set of typical values of the governing parameters, in order to illustrate the convergence behavior of the proposed eigenfunction expansions and inspect the importance of accounting for spatially variable properties in predicting the thermal response of living tissues to external stimulus.
\end{abstract}

(C) 2010 Elsevier Masson SAS. All rights reserved.

\section{Introduction}

The accurate prediction of temperature distributions within living tissues has been a major research topic in the thermal sciences field, in connection with applications such as cryopreservation, thermography for clinical diagnostics, hyperthermia cancer treatments, burn injuries evaluation, and cryosurgery [1]. Pennes' bioheat model has been the most frequently employed equation for the quantitative simulation of the physiological thermal response of living tissues [2], which accounts for the effects of blood circulation and local metabolism in the heat transfer between the more internal tissues and the external environment. A number of traditional analytical and numerical approaches in heat conduction have been applied to the analysis of the bioheat equation, aimed at interpreting the thermal response of tissues, with or without tumors, due to an external stimulation [3-8], but also towards the identification of biological tissues thermophysical properties and

\footnotetext{
* Corresponding author. Tel.: +55 212562 8567; fax: +55 2125628383 .

E-mail address: cotta@mecanica.coppe.ufrj.br (R.M. Cotta).
}

blood perfusion rates in the form of an inverse problem formulation [9-13].

Most available studies are however restricted to situations with uniform effective thermophysical properties across the tissue thickness, which can be fairly heterogeneous in the form of stratified structures. Such simplifications are in part due to the difficulty in analytically handling diffusion problems with space variable coefficients, but also to the additional cumbersome task of estimating the local behavior of the related thermophysical properties. Nevertheless, a few works have addressed the numerical solution of the bioheat equation with space variable coefficients, either due to local histological variations [5] or to the presence of tumor structures embedded within the healthy tissue [13].

Regarding the solution of the direct heat conduction problem for a heterogeneous medium in transient state, a fairly general procedure has been recently advanced [14], based on the Classical Integral Transform Technique (CITT) [15,16]. Ref. [14] dealt with a linear heat conduction problem with space variable coefficients, thus the integral transformation process results into a decoupled transformed ordinary differential system, capable of being analytically solved. Nevertheless, the solution of the corresponding 


\begin{tabular}{|c|c|c|c|}
\hline \multicolumn{2}{|c|}{ Nomenclature } & $T_{f}(x ; t)$ & filter for temperature field \\
\hline$c_{p}(x)$ & space variable specific heat of tissue, eq. (1a) & & \\
\hline$d(x, T)$ & nonlinear dissipation operator coefficient, eq. (1a) & \multicolumn{2}{|c|}{ Greek letters } \\
\hline$h$ & effective heat transfer coefficient, eq. (1a) & $\gamma$ & parameter in property variation function \\
\hline$k(x)$ & space variable thermal conductivity of tissue, eq. (1a) & $\delta$ & coefficient in property variation function \\
\hline$L_{k}$ & individual tissue layer nominal thickness & $\mu$ & eigenvalues of the auxiliary problem, eqs. (5) \\
\hline$L$ & total sample thickness & $\phi(x)$ & property variation function, eqs. (11) \\
\hline$N$ & truncation order in temperature expansion & $\psi$ & eigenfunction of the auxiliary problem \\
\hline M & number of sub-regions in semi-analytical integration & $\rho(x)$ & space variable specific mass of tissue \\
\hline$N_{i}$ & normalization integrals in eigenvalue problem & & \\
\hline$P(x, t)$ & source term, eq. (1a) & \multicolumn{2}{|c|}{ Subscripts and superscripts } \\
\hline$q_{0}(t)$ & applied wall heat flux, eq. (1a) & $i, j$ & order of eigenquantities \\
\hline$q_{m}(x, t)$ & metabolic heat generation & $k$ & index for tissue layer \\
\hline$t$ & time variable & - & integral transform \\
\hline$T(x, t)$ & temperature distribution & $\sim$ & normalized eigenfunction \\
\hline$T_{\infty}(t)$ & external environment temperature & $m$ & index for sub-region in semi-analytical integration \\
\hline$w(x)$ & thermal capacity of tissue, eq. (1a) & & \\
\hline
\end{tabular}

auxiliary eigenvalue problem [14] demands the use of computational methodologies, such as the Generalized Integral Transform Technique (GITT) [16-20]. In the present work we also make use of the GITT, which has already been successfully applied to the solution of bioheat problems with constant coefficients under Pennes' model [21]. However, a hybrid numerical-analytical solution variant is here exploited so as to automatically handle any general functional form of the space variable coefficients, besides the possible nonlinear variation of the blood perfusion rates with temperature, especially during hyperthermia treatments. The analytical nature of the proposed approach permits all manipulations and derivations to be performed exactly in symbolic computation platforms [22].

The methodology here employed was implemented within the mixed symbolic-numerical computational code called UNIT ("UNified Integral Transforms") [23]. The open source UNIT code is a development platform for researchers and engineers interested on hybrid integral transform solutions of convection-diffusion problems, readily available for download from the site http:// 2009unit.vndv.com. Two novel aspects in this approach were here implemented and critically examined, related to the automatic linear filtering and to the semi-analytical evaluation of the integral transformation coefficients.

\section{Problem formulation}

We consider a one-dimensional special case of the general formulation on nonlinear transient diffusion presented and solved in $[16,19]$ by the Generalized Integral Transform Technique (GITT), for the temperature field $T(x, t)$, in the region $x \in[0, L]$. The formulation includes the space variable thermal conductivity and heat capacity of the tissue, as shown in problem (1) below, besides the temperature dependent dissipation term and source function. The coefficients $w(x), d(x, T)$ and $k(x)$, are thus responsible for the information related to the heterogeneity of the medium. The heat conduction equation with the corresponding initial and boundary conditions are given by:

$$
\begin{aligned}
w(x) \frac{\partial T(x, t)}{\partial t}= & \frac{\partial}{\partial x}\left(k(x) \frac{\partial T(x, t)}{\partial x}\right)-d(x, T) T(x, t) \\
& +P(x, t, T), \quad 0<x<L, \quad t>0
\end{aligned}
$$

$$
\begin{aligned}
& T(x, 0)=T_{p}(x), \quad 0<x<L \\
& -\left.k(0) \frac{\partial T(x, t)}{\partial x}\right|_{x=0}=q_{0}(t)+h\left[T_{\infty}(t)-T(0, t)\right], t>0 \\
& \left.\frac{\partial T(x, t)}{\partial x}\right|_{x=L}=0, t>0
\end{aligned}
$$

where,

$$
\begin{aligned}
& w(x)=\rho(x) c_{p}(x) ; \quad d(x, T)=\omega(x, T) \rho_{b} c_{b} \\
& P(x, t, T)=q_{m}(x, t)+\omega(x, T) \rho_{b} c_{b} T_{a}
\end{aligned}
$$

Problem (1) covers a typical one-dimensional transient bioheat problem, such as the one depicted in Fig. 1, based on variable thermophysical properties and blood perfusion rates across the tissue thickness, which can vary more or less abruptly through the transitions of each tissue layer depending on the specific structure. The time variation of the temperature distribution is then promoted by the variation of the applied heat flux at the external tissue surface, $q_{0}(t)$, and eventually also of the external environment temperature, $T_{\infty}(t)$. The exposition of the external tissue face is desirable so as to allow for non-intrusive temperature measurements acquisition such as in infrared thermography. The initial condition, $T_{p}(x)$, may for instance be obtained from the steady-state solution for the situation without external stimulus, i.e., $q_{0}=0$ and constant external environment temperature.

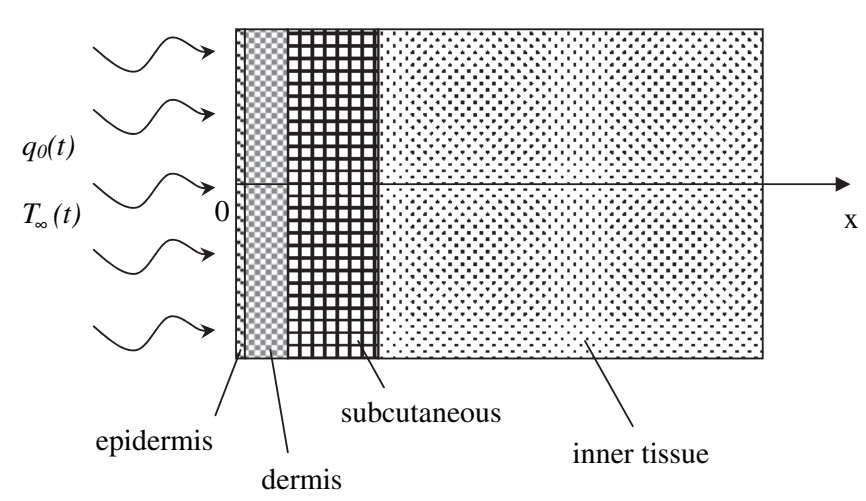

Fig. 1. Schematic diagram of the heterogeneous tissue. 


\section{Integral transform solution}

Before providing the integral transform solution of problem (1), a simple filtering solution is employed for improved convergence behavior of the eigenfunction expansions [16,17], in the form:

$T(x, t)=T_{f}(x ; t)+T^{*}(x, t)$

The simplest possible filtering solution is here adopted, written as a linear function in the space variable that simultaneously satisfies both boundary conditions (1c,d),

$T_{f}(x ; t)=a(t) x+b(t)$

which for the present case results simply in

$T_{f}(x ; t)=b(t) \equiv T_{\infty}(t)+\frac{q_{0}(t)}{h}$

This straightforward linear filter is the default option in the UNIT code [23] and for this reason it is here examined more closely. It essentially homogenizes the boundary conditions in the filtered partial differential problem to be integral transformed. A more complete analytical filter may be preferred [16,17], that further eliminates the source terms in the original equation, eq. (1a), but the above choice was already quite effective in the present situation. The filtered temperature problem formulation is then given by:

$\begin{aligned} w(x) \frac{\partial T^{*}(x, t)}{\partial t}= & \frac{\partial}{\partial x}\left(k(x) \frac{\partial T^{*}(x, t)}{\partial x}\right)-d(x, T) T^{*}(x, t) \\ & +P^{*}(x, t, T), \quad 0<x<L, \quad t>0\end{aligned}$

$T^{*}(x, 0)=f(x) \equiv T_{p}(x)-T_{f}(x ; 0)$

$-\left.k(0) \frac{\partial T^{*}(x, t)}{\partial x}\right|_{x=0}+h T^{*}(0, t)=0, \quad t>0$

$\left.\frac{\partial T^{*}(x, t)}{\partial x}\right|_{x=L}=0, t>0$

where the filtered source term is written as

$$
\begin{aligned}
P^{*}(x, t, T)= & q_{m}(x, t)+\omega(x, T) \rho_{b} c_{b}\left[T_{a}-T_{f}(x ; t)\right] \\
& -w(x) \frac{\partial T_{f}(x ; t)}{\partial t}
\end{aligned}
$$

The formal solution of problem (3) is then obtained with the Generalized Integral Transform Technique [16-19], and is written as the inverse formula below:

$T^{*}(x, t)=\sum_{i=1}^{\infty} \tilde{\psi}_{i}(x) \bar{T}_{i}(t)$

where the transformed potentials are defined with the integral transformation operation given by

$\bar{T}_{i}(t)=\int_{0}^{L} w^{*}(x) \tilde{\psi}_{i}(x) T^{*}(x, t) d x$

The eigenvalues $\mu_{i}$ and eigenfunctions $\psi_{i}(x)$, are obtained from the chosen eigenvalue problem below:

$\frac{d}{d x}\left[k^{*}(x) \frac{d \psi_{i}(x)}{d x}\right]+\left[\mu_{i}^{2} w^{*}(x)-d^{*}(x)\right] \psi_{i}(x)=0, \quad x \in[0, L]$

with boundary conditions

$-k^{*}(0) \frac{d \psi_{i}(x)}{d x}+h^{*} \psi_{i}(x)=0, \quad x=0$ $\frac{d \psi_{i}(x)}{d x}=0, \quad x=L$

where the coefficients $k^{*}(x), w^{*}(x)$, and $d^{*}(x)$ are simpler coefficients chosen so as to construct an eigenvalue problem of known analytical solution to offer the basis for the eigenfunction expansion. For convenience, we also require that eq. (5b) matches the homogeneous version of the filtered boundary condition, eq. (3c), by letting $h^{*}=h k^{*}(0) / k(0)$. The other quantities that appear in the inverse formula (4a) are computed after solving problem (5), such as:

$\tilde{\psi}_{i}(x)=\frac{\psi_{i}(x)}{\sqrt{N_{i}}}$, normalized eigenfunctions

$N_{i}=\int_{0}^{L} w^{*}(x) \psi_{i}^{2}(x) d x$, normalization integrals

Before proceeding to the integral transformation process itself, it is of interest to avoid an implicit transformed system, and thus eq. (5a) can be rewritten in terms of the chosen weighting function $w^{*}(x)$, as follows:

$w^{*}(x) \frac{\partial T^{*}(x, t)}{\partial t}=\frac{w^{*}(x)}{w(x)}\left[\frac{\partial}{\partial x}\left(k(x) \frac{\partial T^{*}(x, t)}{\partial x}\right)-d(x, T) T^{*}(x, t)\right.$

or simply,

$$
\left.+P^{*}(x, t, T)\right], \quad 0<x<L, t>0
$$

$w^{*}(x) \frac{\partial T^{*}(x, t)}{\partial t}=G(x, t, T), \quad 0<x<L, \quad t>0$

with,

$G(x, t, T)=\frac{w^{*}(x)}{w(x)}\left[\frac{\partial}{\partial x}\left(k(x) \frac{\partial T^{*}(x, t)}{\partial x}\right)-d(x, T) T^{*}(x, t)+P^{*}(x, t, T)\right]$

The integral transformation is now performed by operating eq. (7b) on with $\int_{0}^{L} \tilde{\psi}_{i}(x)-d x$, to yield the following transformed ordinary differential system:

$\frac{d \bar{T}_{i}(t)}{d t}=\bar{g}_{i}\left(t, \bar{T}_{j}\right), \quad t>0, \quad i, j=1,2, \ldots$

with the transformed source terms given by

$\bar{g}_{i}\left(t, \bar{T}_{j}\right)=\int_{0}^{L} G(x, t, T) \tilde{\psi}_{i}(x) d x$

and the transformed initial conditions, after operating eq. (3b) on with $\int_{0}^{L} w^{*}(x) \tilde{\psi}_{i}(x)-d x$,

$\bar{f}_{i}=\int_{0}^{L} w^{*}(x) f(x) \tilde{\psi}_{i}(x) d x$

The ODE system (8) can be numerically solved to provide results for the transformed temperatures, upon truncation to a sufficiently large finite order $N$, which will be combined by the inverse formula (4a) to provide the desired original temperature field.

\section{Computational algorithm: the UNIT code}

The constructed UNIT code in the Mathematica 7.0 platform [23] encompasses all of the symbolic derivations that are required in the 
above GITT formal solution, besides the numerical computations that are required in the solution of the chosen eigenvalue problem and the transformed ODE system. The user essentially needs to specify the problem formulation, according to Eqs. (1), and then choose how to present results according to the specific needs.

In order to computationally solve the problem defined by Eqs. (1), a quite straightforward general algorithm can be described as follows:

- The user provides the input and problem formulation module, which includes the equation and boundary condition coefficients in eqs. (1), besides the corresponding source terms. There is an implicit choice of eigenvalue problem when the coefficients $k^{*}(x), w^{*}(x)$, and $d^{*}(x)$ are specified so as to form the auxiliary problem to be solved for.

- The automatic filtering module is then activated, which is either the simplest possible choice of functions that essentially satisfy the boundary conditions, to make them homogeneous as here discussed, or the filter is provided as a problem formulation by the user, to be handled via symbolic or numerical computation. The option of not providing a filtering solution is also allowed for, either because it might not be actually necessary or as a solution strategy to be complemented by an integral balance acceleration a posteriori [16,17].

- The auxiliary eigenvalue problem of Eqs. (5) is solved for the eigenvalues and related normalized eigenfunctions, either in analytic explicit form, when applicable, as obtained by the symbolic routine DSolve [22], after separation of variables in multidimensional applications, or through the GITT itself $[14,17,20]$.

- The transformed initial condition is computed, either analytically (function Integrate [22]) or with a general-purpose procedure through adaptive numerical integration (function NIntegrate [22]). Two additional options are provided to the user, namely, a semi-analytical evaluation where the analytical integration of the eigenfunction oscillatory behavior is preserved [23], and a simplified and cost-effective numerical integration with Gaussian quadrature, automatically exploiting the frequency of oscillation of the eigenfunctions in the choice of intervals. Similarly, the coefficients on the transformed O.D.E. system of Eq. (8a), once they are not dependent on the transformed potentials, can be evaluated in advance. For the more general situation of nonlinear coefficients, there are some computational savings in grouping them into a single integrand, as represented in Eq. (8b). The coefficients in the transformed system can be obtained by analytical integration, if feasible, or again by the automatic Gaussian quadrature scheme that accounts for the knowledge on the eigenfunctions oscillatory behavior. The alternative semi-analytical integration procedure is also implemented, which is particularly convenient in nonlinear formulations that might require costly numerical integration. For instance, the integral transformation of the equation source term for homogeneous filtered boundary conditions would then be evaluated as:

$$
\begin{aligned}
\bar{g}_{i}\left(t, \bar{T}_{j}\right) & =\int_{v} \tilde{\psi}_{i}(x) G(x, t, T) d v \\
& =\sum_{m=1}^{M} \int_{v_{m}} \tilde{\psi}_{i}(x) \widehat{G}_{m}(x, t, T) d v
\end{aligned}
$$

where $\widehat{G}_{m}(x, t, \mathrm{~T})$ are simpler representations of the source term, defined in $M$ sub-regions $V_{m}$, for which analytical integration of the eigenfunctions is still obtainable. The simplest choice would be the adoption of uniform values of the source terms within the subdomains (zeroth order approximation), but linear and quadratic representations of the source terms behavior are also implemented [23].

- The truncated O.D.E. system of Eqs. (8a) and (8c) is then numerically solved through function NDSolve of the Mathematica system [22]. In general, such initial value problem solvers should work under the automatic selection of a stiff system situation, such as with the BDF (Gear's) method [22], since the resulting system is likely to become stiff, especially for increasing truncation orders. This subroutine offers an interesting combination of accuracy control, simplicity in use, and reliability.

- Once all the intermediate numerical tasks are accomplished within user-prescribed accuracy, one is left with the need of reaching convergence in the eigenfunction expansions and controlling the truncation order $N$ for the requested accuracy in the final solution. The analytic nature of the inversion formula allows for a direct testing procedure at each specified position within the medium where a solution is desired, and the truncation order $N$ can be decreased (or eventually increased), to fit the user global error requirements over the entire solution domain. The simple tolerance testing formula employed is written as

$$
\varepsilon(t)=\max \left|\frac{\sum_{i=N^{*}+1}^{N} \tilde{\psi}_{i}(x) \bar{T}_{i}(t)}{T_{f}(x ; t)+\sum_{i=1}^{N} \tilde{\psi}_{i}(x) \bar{T}_{i}(t)}\right|
$$

The numerator in Eq. (10) represents those terms that in principle might be abandoned in the evaluation of the inverse formula, without disturbing the final result to within the userrequested accuracy target. Therefore, this testing can be implemented by choosing the value of $N^{*}$ in the numerator sum, then offering error estimations at any of the selected test positions within the domain.

\section{Results and discussion}

The bioheat problem solution here illustrated involves the analysis of fairly abrupt transitions of tissue layers, as depicted in Fig. 1, to challenge the algorithm, which includes the epidermis, the dermis, the subcutaneous tissue, and the inner tissue. The corresponding data for the results to be presented were extracted from ref. [5] and are reproduced in Table 1 . The epidermis layer was disregarded in light of its negligible thickness ( $<80$ microns) and relatively slight thermal influence in the test case to be here considered.

For the sake of illustration, the space variable distribution for each thermophysical property is here considered to be governed by the parameter $\gamma_{k}$ in the function below:

$$
\begin{aligned}
& \phi(x)=\prod_{k=1}^{N_{k}} \phi_{k-1}+\left(\phi_{k}-\phi_{k-1}\right) \delta_{k}(x) \\
& \delta_{k}(x)=\frac{1}{1+e^{-\gamma_{k} \frac{\left(x-x_{k}\right)}{L}}}
\end{aligned}
$$

with $x_{k}$ being the assumed reference transition position between two adjacent tissue layers. Fig. 2a,b illustrates the behavior of the thermophysical properties across the full tissue thickness for the selected test case, where Fig. 2a refers to the thermal conductivity and Fig. $2 b$ represents the thermal capacity variation. Fig. 3a presents the imposed external stimulus, in the form of a wall 
Table 1

Parameters values used in bioheat equation simulation [5].

\begin{tabular}{|c|c|}
\hline Sample total thickness & $L=0.042 \mathrm{~m}$ \\
\hline Metabolic heat generation & $q_{m}=420 \mathrm{~W} / \mathrm{m}^{3}$ \\
\hline \multirow[t]{3}{*}{ Arterial blood properties } & $\rho_{b}=1060 \mathrm{~kg} / \mathrm{m}^{3}$ \\
\hline & $c_{b}=3770 \mathrm{~J} / \mathrm{kgC}$ \\
\hline & $T_{\mathrm{a}}=37^{\circ} \mathrm{C}$ \\
\hline \multirow[t]{4}{*}{ Dermis properties } & $\rho=1200 \mathrm{~kg} / \mathrm{m}^{3}$ \\
\hline & $c_{p}=3300 \mathrm{~J} / \mathrm{kgC}$ \\
\hline & $k=0.45 \mathrm{~W} / \mathrm{mC}$ \\
\hline & $\begin{array}{l}l=0.002 \mathrm{~m} \\
\omega=0.00125 \mathrm{~m}^{3} / \mathrm{s} \mathrm{m}^{3} \text { tissue }\end{array}$ \\
\hline \multirow[t]{5}{*}{ Subcutaneous tissue properties } & $\rho=1000 \mathrm{~kg} / \mathrm{m}^{3}$ \\
\hline & $c_{p}=2500 \mathrm{~J} / \mathrm{kgC}$ \\
\hline & $k=0.19 \mathrm{~W} / \mathrm{mC}$ \\
\hline & $l=0.01 \mathrm{~m}$ \\
\hline & $\omega=0.00125 \mathrm{~m}^{3} / \mathrm{s} \mathrm{m}^{3}$ tissue \\
\hline \multirow[t]{5}{*}{ Inner tissue properties } & $\rho=1000 \mathrm{~kg} / \mathrm{m}^{3}$ \\
\hline & $c_{p}=4000 \mathrm{~J} / \mathrm{kgC}$ \\
\hline & $k=0.5 \mathrm{~W} / \mathrm{mC}$ \\
\hline & $l=0.03 \mathrm{~m}$ \\
\hline & $\omega=0.00125 \mathrm{~m}^{3} / \mathrm{s} \mathrm{m}^{3}$ tissue \\
\hline \multirow[t]{2}{*}{ Parameters in thermal properties function } & $\gamma_{k}=200$ \\
\hline & $x_{c}=x_{k}$ \\
\hline Effective heat transfer coefficient & $h=10 \mathrm{~W} / \mathrm{m}^{2} \mathrm{C}$ \\
\hline \multirow[t]{3}{*}{ Parameters in applied heat flux function } & $\gamma_{t}=200$ \\
\hline & $x_{t}=10$ and $110 \mathrm{~s}$ \\
\hline & $q_{0}=1000 \mathrm{~W} / \mathrm{m}^{2}$ \\
\hline Ambient temperature & $T_{\infty}=20{ }^{\circ} \mathrm{C}$ \\
\hline
\end{tabular}

applied heat flux, with steep turning on and off periods, such as achieved through the activation of flash lamps over the external tissue surface. Fig. $3 b$ then shows the temperature steady-state solution achieved for the data in Table 1 when the external stimulus is not present, which is in fact the initial condition for the present transient problem.
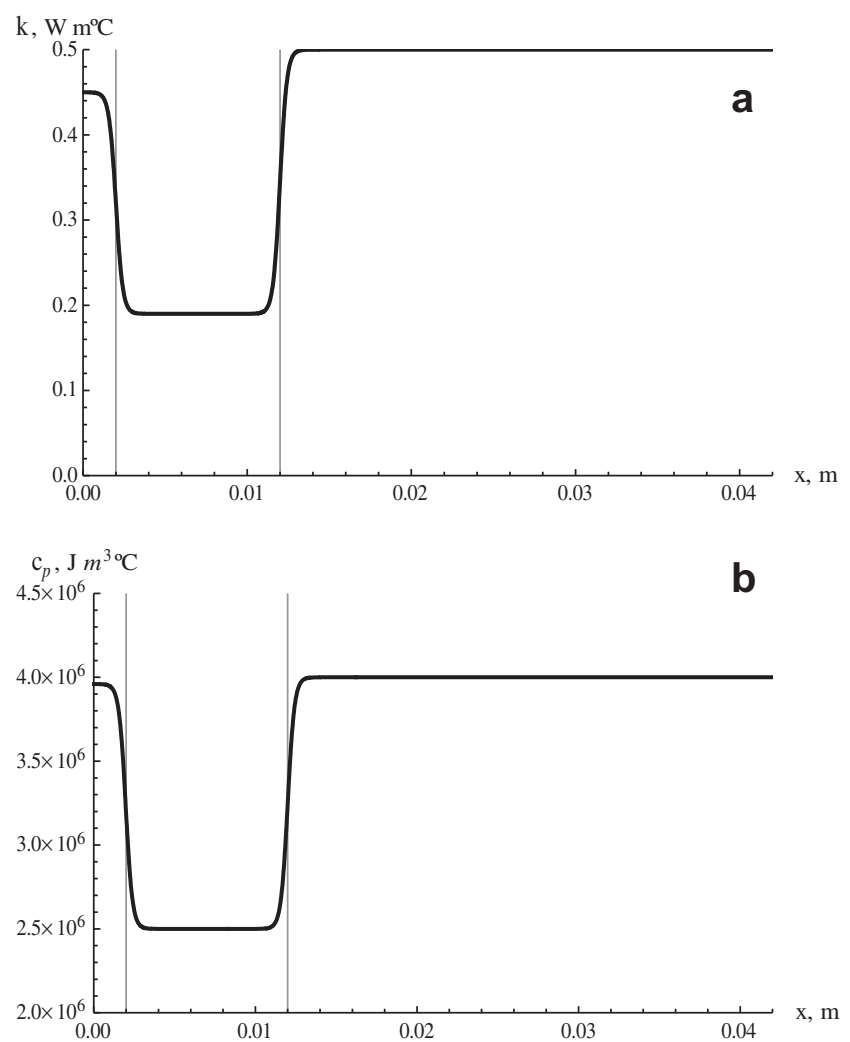

Fig. 2. Spatial variation of the (a) thermal conductivity and of the (b) thermal capacity, according to data in Table 1.
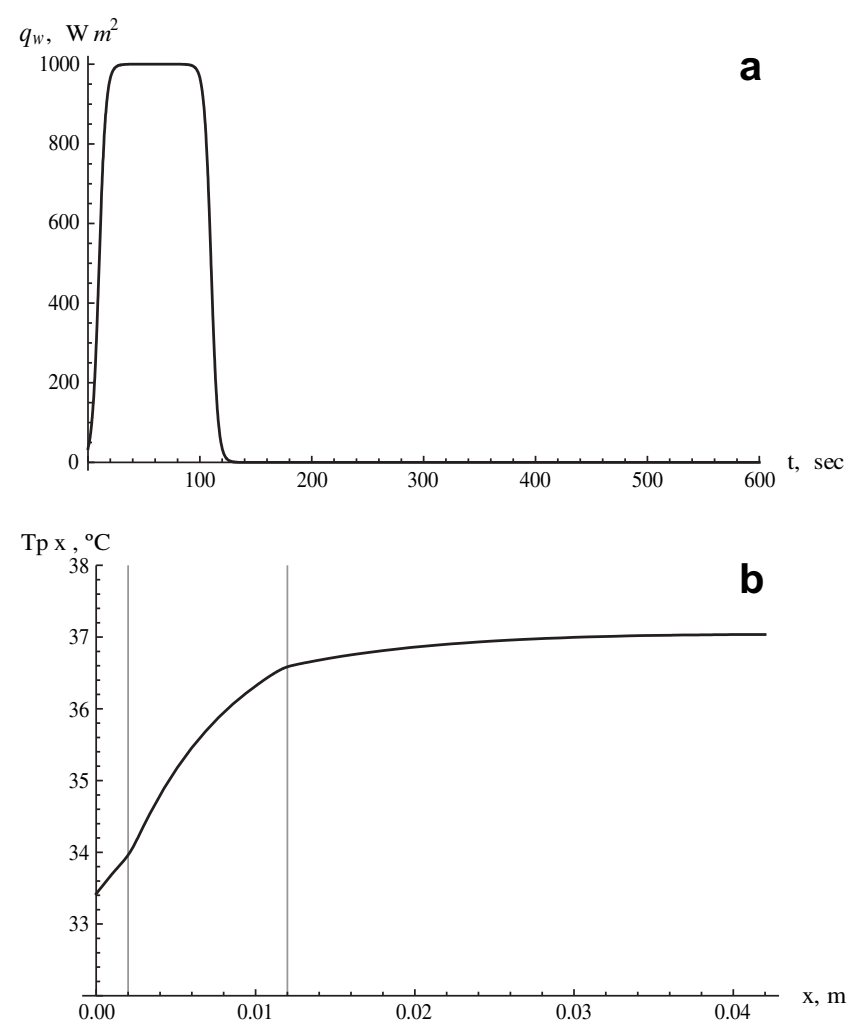

Fig. 3. Adopted distributions of the (a) prescribed wall heat flux (external stimulus) and of the (b) initial temperature condition (steady-state solution for $q_{0}=0$ ).

This direct problem solution was obtained through the Generalized Integral Transform Technique as previously described. The simplest possible choice of coefficients in the auxiliary eigenvalue problem was made, with $w^{*}(x)=k^{*}(x)=1$ and $d^{*}(x)=0$, so as to challenge the convergence behavior of the fairly simple set of eigenfunctions thus adopted. Also, only the automatic linear filtering option was activated in the code implementation, not to enhance convergence with extra analytical information that accounts, even though partially, for the equation source term. A thorough convergence analysis was performed to illustrate the required number of terms in the temperature expansion, as well as the number of regions for the semi-analytical integration. For instance, Tables $2 a$ and $b$ present a set of results for the temperature distribution across the tissue, at the time values $t=50$ and $100 \mathrm{~s}$, respectively, as we increase the truncation order in the eigenfunction expansion, from $N=10$ up to 60 . Also, the last column provides some numerical results for comparison purposes, obtained with the Method of Lines

Table 2a

Illustration of convergence behavior of temperature field eigenfunction expansion $(t=50 \mathrm{~s})$.

\begin{tabular}{llllllll}
\hline$x$ & $N=10$ & $N=20$ & $N=30$ & $N=40$ & $N=50$ & $N=60$ & Numer. $^{\text {a }}$ \\
\hline 0. & 38.897 & 38.841 & 38.774 & 38.784 & 38.796 & 38.793 & 38.836 \\
0.0042 & 35.363 & 35.341 & 35.315 & 35.321 & 35.326 & 35.325 & 35.330 \\
0.0084 & 36.046 & 36.038 & 36.037 & 36.032 & 36.033 & 36.034 & 36.033 \\
0.0126 & 36.603 & 36.626 & 36.619 & 36.6183 & 36.619 & 36.619 & 36.618 \\
0.0168 & 36.782 & 36.784 & 36.778 & 36.776 & 36.777 & 36.777 & 36.777 \\
0.021 & 36.876 & 36.887 & 36.880 & 36.880 & 36.881 & 36.881 & 36.881 \\
0.0252 & 36.952 & 36.954 & 36.948 & 36.947 & 36.948 & 36.948 & 36.948 \\
0.0294 & 36.986 & 36.996 & 36.990 & 36.990 & 36.991 & 36.991 & 36.991 \\
0.0336 & 37.021 & 37.022 & 37.017 & 37.016 & 37.016 & 37.017 & 37.017 \\
0.0378 & 37.026 & 37.036 & 37.030 & 37.030 & 37.031 & 37.031 & 37.031 \\
0.042 & 37.040 & 37.041 & 37.035 & 37.034 & 37.035 & 37.035 & 37.035 \\
\hline
\end{tabular}

${ }^{a}$ NDSolve routine of Mathematica system [22]. 
Table 2b

Illustration of convergence behavior of temperature field eigenfunction expansion $(t=100 \mathrm{~s})$

\begin{tabular}{llllllll}
\hline$x$ & $N=10$ & $N=20$ & $N=30$ & $N=40$ & $N=50$ & $N=60$ & Numer. $^{a}$ \\
\hline 0. & 41.904 & 41.825 & 41.734 & 41.759 & 41.776 & 41.769 & 41.829 \\
0.0042 & 36.908 & 36.831 & 36.786 & 36.791 & 36.800 & 36.799 & 36.813 \\
0.0084 & 36.115 & 36.169 & 36.162 & 36.156 & 36.157 & 36.159 & 36.159 \\
0.0126 & 36.645 & 36.633 & 36.621 & 36.620 & 36.621 & 36.621 & 36.621 \\
0.0168 & 36.752 & 36.788 & 36.777 & 36.776 & 36.777 & 36.777 & 36.777 \\
0.021 & 36.902 & 36.891 & 36.880 & 36.879 & 36.881 & 36.881 & 36.881 \\
0.0252 & 36.929 & 36.957 & 36.948 & 36.946 & 36.948 & 36.948 & 36.948 \\
0.0294 & 37.008 & 37.000 & 36.990 & 36.989 & 36.991 & 36.991 & 36.991 \\
0.0336 & 37.000 & 37.026 & 37.017 & 37.015 & 37.017 & 37.017 & 37.017 \\
0.0378 & 37.047 & 37.040 & 37.030 & 37.029 & 37.031 & 37.031 & 37.031 \\
0.042 & 37.018 & 37.044 & 37.035 & 37.033 & 37.035 & 37.035 & 37.035 \\
\hline
\end{tabular}

a NDSolve routine of Mathematica system [22].

implemented in the routine NDSolve of the Mathematica system, with extra mesh refinement than in the default usage. Clearly, from the GITT results one may observe that in the worst situation we have already achieved at least four fully converged significant digits in the temperature profile, throughout the medium and for two time values within the applied wall heat flux variation period. Also, convergence is apparently slightly slower at the boundary $x=0$. The provided numerical solution is quite adherent to the fully converged GITT solution, recovering the first three significant digits in the worst situation, which is again at the points in the vicinity of the external boundary.

Fig. 4a,b illustrates the transient behavior of the temperature field across the tissue thickness for (a) full tissue thickness $(0<x<L)$ and for (b) the detailed view of the first two layers. Here, the increasingly long dashes correspond to decreasing values of
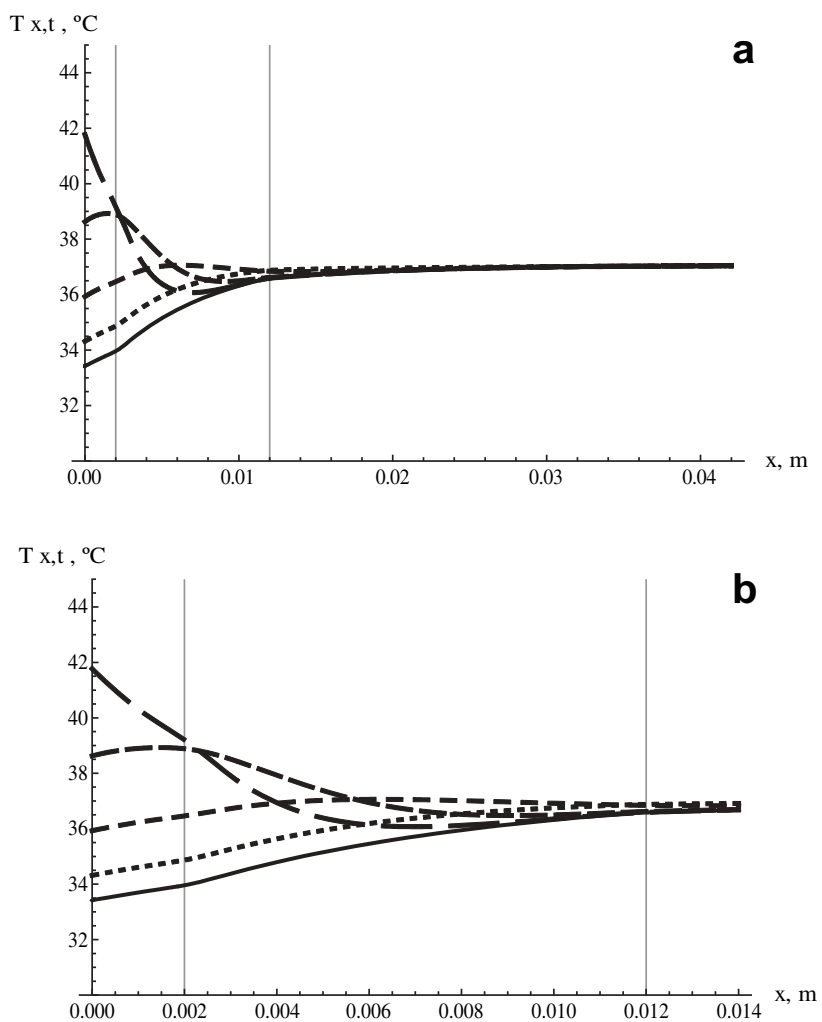

Fig. 4. Transient behavior of the temperature field across the tissue thickness for (a) full tissue thickness $(0<x<L)$ and of the (b) detailed view of the first two layers. (Solid line - initial condition, $t=0$, very long dashes $-t=100 \mathrm{~s}$, long dashes $t=150 \mathrm{~s}$, medium dashes $-t=300 \mathrm{~s}$, small dashes $-t=600 \mathrm{~s}$.)

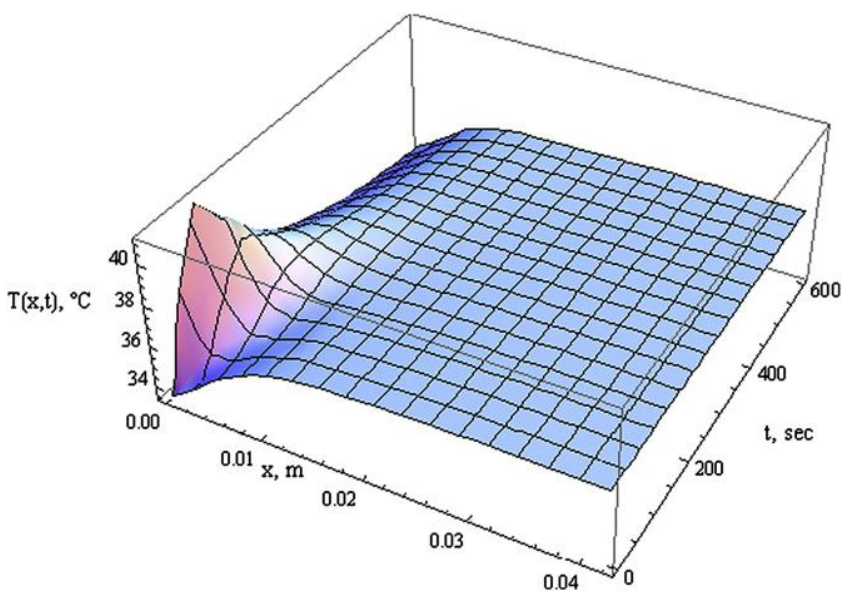

Fig. 5. Converged results $(N=60)$ obtained with GITT for the temperature field along both the space and time variables.

time (solid line - initial condition, $t=0$, very long dashes $t=100 \mathrm{~s}$, long dashes $-t=150 \mathrm{~s}$, medium dashes $-t=300 \mathrm{~s}$, small dashes $-t=600 \mathrm{~s}$ ). It is clear that the surface region responds quite rapidly to the external stimulus, and that this information is markedly dumped within the first two layers of the tissue structure, thus leaving the third tissue layer practically unaffected. This behavior is more clearly observable in Fig. 5 below, for the threedimensional plot of the temperature field against space coordinate and time, where the relatively small thermally affected region is noticeable, for the present experimental conditions.
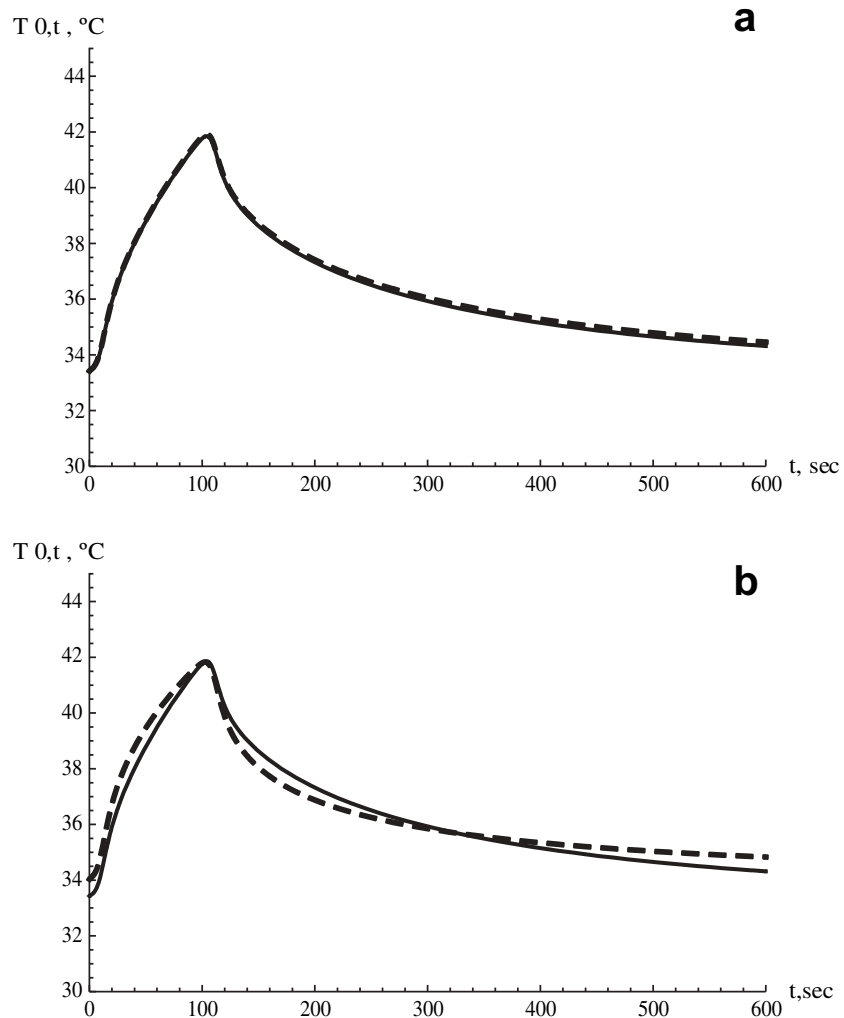

Fig. 6. Converged results $(N=60)$ obtained with GITT for the external tissue temperature evolution (a) for variable properties, GITT (solid) and numerical solution via NDSolve [22] (dashed); (b) for both variable properties (solid) and averaged uniform properties (dashed). 


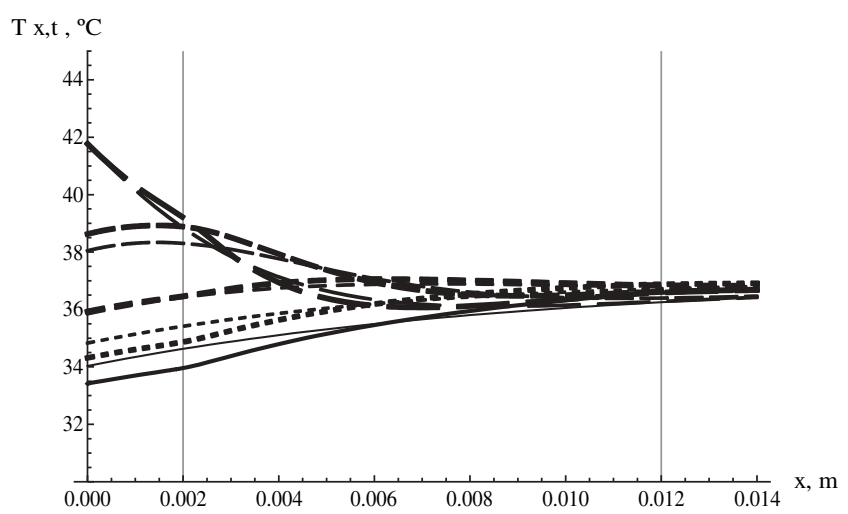

Fig. 7. Transient behavior of the temperature field across the tissue thickness for a detailed view of the first two layers, with variable properties (thicker lines) and uniform averaged properties (thinner lines). (Solid line - initial condition, $t=0$, very long dashes $-t=100 \mathrm{~s}$, long dashes $-t=150 \mathrm{~s}$, medium dashes $-t=300 \mathrm{~s}$, small dashes $-t=600 \mathrm{~s}$.)

Fig. 6a provides a comparison of the external tissue surface temperature evolution, as obtained through GITT with $N=60$ terms (solid line) and through the numerical method implemented in routine NDSolve [22] (dashed line), with sufficient mesh refinement. The overall agreement is indeed very good, with slightly noticeable deviations for regions closer to the internal tissue end. Finally, Fig. 6b again shows the fully converged results for the external tissue temperature, but now for either variable properties (solid line) or for uniform averaged thermophysical properties (dashed line), as estimated from integration of the profiles in Fig. 2. Clearly, more significant deviations are now observable in the external temperature behavior, which may already be sensed in a typical thermographic measurement system. Fig. 7 then compares the temperature distribution across the first two layers of the tissue, again for both the variable properties model (thick lines) and the uniform averaged properties approximation (thin lines). The deviations within the medium for the two models results is even more significant, confirming the interest in the more accurate determination of the variable thermophysical properties functional dependence and on the appropriate representation of the thermal phenomena by accounting for this variability.

The results obtained in this work reveal that the proposed approach can provide accurate estimation of the temperature distributions in bioheat transfer problems and is robust and capable of providing accurate results even for fairly abrupt variations of the thermophysical properties variations. Nonlinear functional behavior of the perfusion rates, such as in hyperthermia treatment, may also be directly accounted for and computed via integral transforms.

\section{Acknowledgements}

The authors would like to acknowledge the financial support provided by $\mathrm{CNPq}$, Brazilian agency for the fostering of sciences. This work is dedicated in memoriam to both Dr. Bianca Pires Cotta,
M.D. (24/04/1984-31/05/2009), our beloved daughter, and her advisor and uncle Prof. Gerson Cotta Pereira (18/08/1942-03/01/ 2010). This research was initiated in 2009, a few months before Bianca, and later on Gerson, left us. Nevertheless, their enthusiasm will live forever and will provide us with the strength to pursue it further.

\section{References}

[1] K.R. Diller, J.W. Valvano, J.A. Pearce, Bioheat transfer. in: F. Kreith (Ed.), The CRC Handbook of Thermal Engineering. CRC Press, Boca Raton, FL, 2000 (Section 4.4).

[2] H.H. Pennes, Analysis of tissue and arterial blood temperatures in the resting human forearm. J. Appl. Physiol. 1 (1948) 93-122.

[3] J. Liu, L.X. Xu, Boundary information based diagnostics on the thermal states of biological bodies. Int. J. Heat Mass Transf. 43 (2000) 2827-2839.

[4] Z.S. Deng, J. Liu, Blood perfusion based model for characterizing the temperature fluctuation in living tissues. Physica A 300 (2001) 521-530.

[5] S.C. Jiang, N. Ma, H.J. Li, X.X. Zhang, Effects of thermal properties and geometrical dimensions on skin burn injuries. Burns 28 (2002) 713-717.

[6] T.C. Shih, P. Yuan, W.L. Lin, H.S. Kou, Analytical analysis of the Pennes bioheat transfer equation with sinusoidal heat flux condition on skin surface. Med. Eng. Phys. 29 (2007) 946-953.

[7] S. Maruyama, J. Okajima, A. Komiya, H. Takeda, Estimation of temperature distribution in biological tissue by using solutions of bioheat transfer equation. Heat Transf. Asian Res. 37 (6) (2008) 374-386.

[8] J. Sun, A. Zhang, L.X. Xu, Evaluation of alternate cooling and heating for tumor treatment. Int. J. Heat Mass Transf. 51 (2008) 5478-5485.

[9] J.S. Peng, Y.Q. Xia, T. Gao, X.L. Lei, S.Y. Zhao, A surface heat disturbance method for measuring local tissue blood perfusion rate. Heat Transf. Asian Res. 29 (1) (2000) 34-44

[10] K. Yue, X. Zhang, F. Yu, Simultaneous estimation of thermal properties of living tissues using noninvasive method. Int. J. Thermophys. 28 (5) (2007) 1470-1489.

[11] C.H. Huang, C.Y. Huang, An inverse problem in estimating simultaneously the effective thermal conductivity and volumetric heat capacity of biologica tissue. Appl. Math. Model. 31 (2007) 1785-1797.

[12] D. Trucu, D.B. Ingham, D. Lesnic, An inverse coefficient identification problem for the bio-heat equation. Inverse Probl. Sci. Eng. 17 (1) (2009) 65-83.

[13] P.W. Partridge, L.C. Wrobel, A coupled dual reciprocity BEM/genetic algorithm for identification of blood perfusion parameters. Int. J. Num. Methods Heat Fluid Flow 19 (1) (2009) 25-38.

[14] C.P. Naveira-Cotta, R.M. Cotta, H.R.B. Orlande, O. Fudym, Eigenfunction expansions for transient diffusion in heterogeneous media. Int. J. Heat Mass Transf. 52 (2009) 5029-5039.

[15] M.D. Mikhailov, M.N. Ozisik, Unified Analysis and Solutions of Heat and Mass Diffusion. John Wiley, NY, 1984, also, Dover Publications, 1994.

[16] R.M. Cotta, Integral Transforms in Computational Heat and Fluid Flow. CRC Press, USA, 1993.

[17] R.M. Cotta, M.D. Mikhailov, Heat Conduction: Lumped Analysis, Integral Transforms, Symbolic Computation. Wiley-Interscience, NY, 1997.

[18] R.M. Cotta, The Integral Transform Method in Thermal and Fluids Sciences and Engineering. Begell House, New York, 1998.

19] R.M. Cotta, M.D. Mikhailov, Hybrid methods and symbolic computations. in W.J. Minkowycz, E.M. Sparrow, J.Y. Murthy (Eds.), Handbook of Numerical Heat Transfer, second ed. Wiley, New York, 2006, pp. 493-522.

[20] M.D. Mikhailov, R.M. Cotta, Integral transform method for eigenvalue problems. Commun. Num. Methods Eng. 10 (1994) 827-835.

[21] A.V. Presgrave, R.O.C. Guedes, F. Scofano Neto, Integral transform solution to the endometrial ablation problem. J. Braz. Soc. Mech. Sci. Eng. 31 (2009) 117-124.

[22] S. Wolfram, The Mathematica Book, Version 5.2. Cambridge-Wolfram Media, 2005.

[23] L.A. Sphaier, R.M. Cotta, C.P. Naveira-Cotta, J.N.N. Quaresma, The UNIT (Unified Integral Transforms) Symbolic-Numerical Computational Platform for Benchmarks in Convection-Diffusion Problems. 30 CILAMCE - Iberian-Latin-American Congress on Computational Methods in Engineering, Armação de Búzios, RJ, November 2009. 